\title{
AVALIAÇÃO DA CAMADA NITRETADA POR DESCARGAS ELÉTRICAS NA LIGA DE TITÂNIO Ti6AI4V*
}

\author{
Rafael Morais Ataíde Murta ${ }^{1}$ \\ Ernane Rodrigues da Silva ${ }^{2}$ \\ Rogério Felício dos Santos ${ }^{3}$ \\ Steferson Ferreira de Oliveira ${ }^{4}$
}

\section{Resumo}

Desenvolvido nos últimos anos, o processo NDE, vem se tornando uma maneira promissora de usinar e nitretar simultânea a superfície de materiais metálicos. A Usinagem por Descargas Elétricas (EDM) é um processo de usinagem não convencional que tem grande potencial em aplicações de engenharia de superfície, ao melhorar a resistência superficial do material por meio de tratamentos termoquímicos, como por exemplo, a nitretação. Propriedades como: baixa densidade, boa resistência à tração (comparável à de muitos aços ligados) e excelente resistência à corrosão, possibilitam a aplicação do titânio e suas ligas nas indústrias aeroespacial, naval, química e automotiva e ainda no campo da medicina. Porém, raramente são usados como peças sujeitas ao deslizamento, devido a sua devido à sua baixa resistência aos mecanismos de desgaste abrasivo e adesivo e alto coeficiente de atrito. O tratamento termoquímico de nitretação pode solucionar esta restrição. Além de aumentar a resistência ao desgaste, o nitreto de titânio melhora a biocompatibilidade de suas ligas. O objetivo deste trabalho foi estudar a camada nitretada na liga de titânio Ti6Al4V por meio do processo de Nitretação por Descargas Elétricas (NDE), usando como fluído dielétrico solução de água deionizada e ureia granulada, como fonte de nitrogênio. Uma máquina de EDM por penetração foi utilizada no processo. Cobre e grafite foram utilizados como materiais dos eletrodos ferramenta. Foi avaliada a usinabilidade da liga Ti6Al4V com solução de água e ureia, relacionada aos parâmetros: taxa de remoção de material (TRM), taxa de desgaste (TD) e desgaste volumétrico relativo (DVR) para eletrodo de cobre e de grafite. Para caracterizar a camada nitretada foram realizadas microscopia óptica e medição de microdureza Vickers. A formação de nitretos na superfície foi avaliada por meio de difração de raio-x. Imagens metalográficas da seção transversal da superfície mostraram a formação da camada nitretada, devido ao ganho no valor da dureza. Os parâmetros relacionados à usinagem apresentaram valores que indicam um melhor desempenho quando se usa eletrodo ferramenta de cobre em relação à grafita. Nitretos de titânio foram observados no difratogramas da amostras nitretadas por NDE.

Palavras-chave: EDM; Nitretação por descargas elétricas; Implantação iônica; Nitretos de titânio, Liga Ti6A/4V.

\section{EVALUATION OF NITRATE LAYER BY ELECTRIC DISCHARGE ON TITANIUM ALLOY TIGAI4V}

\section{Abstract}

Developed in recent years, the process EDN has become a promising way to simultaneously process and nitride the surface of metallic materials. EDM is an unconventional machining process that has great potential in surface engineering applications by improving the surface resistance of the material by means of thermochemical treatments such as nitriding. Properties such as low density, good tensile strength (comparable to many bonded steels) and excellent corrosion resistance, allow the application of titanium and its alloys in the aerospace, naval, chemical and automotive industries and in the medical field. However, they are rarely used as sliding parts because of their low resistance to abrasive and adhesive wear mechanisms and high coefficient of friction. The thermochemical treatment of nitriding can solve this restriction. In addition to increasing wear resistance, titanium nitride improves the biocompatibility of its alloys. The 
objective of this work was to study the nitrided layer in the Ti6Al4V titanium alloy by means of the Electric Discharge Nitriding (EDN) process, using a solution of deionized water and granulated urea as a source of nitrogen as the dielectric fluid. A Penetrating Electric Discharge Machining (EDM) machine was used in the process. Copper and graphite were used as materials of the electrodes tool. The machinability of Ti6Al4V alloy with water and urea solution, related to the parameters: material removal rate (MRR), wear rate (WR) and relative volumetric wear (RVW) for copper and graphite electrodes were evaluated. To characterize the nitrided layer were performed optical microscopy and Vickers microhardness measurement. The formation of nitrides on the surface was evaluated by means of x-ray diffraction. Metallographic images of the cross section of the surface showed the formation of the nitrided layer, due to the gain in the hardness value. The parameters related to the machining presented values that indicate a better performance when using copper tool electrode in relation to the graphite. Titanium nitrides were observed in the diffractograms of nitrided samples by EDN.

Keywords: EDM; Electric Discharge Nitriding; Ion implantion; Titanium nitrides, Alloy Ti6Al4V.

1 Engenheiro Mecânico, Mestrando, Departamento de Engenharia de Materiais, Centro Federal de Educação Tecnológica de Minas Gerais, Belo Horizonte, Minas Gerais, Brasil.

2 Matemático, Doutor em Engenharia Mecânica, Professor, Departamento de Engenharia de Materiais, Centro Federal de Educação Tecnológica de Minas Gerais, Belo Horizonte, Minas Gerais, Brasil.

3 Engenheiro Mecânico, Doutor em Engenharia Mecânica, Departamento de Engenharia de Materiais, Centro Federal de Educação Tecnológica de Minas Gerais, Belo Horizonte, Minas Gerais, Brasil.

4 Engenheiro Metalurgista, Mestrando, Bolsista, Departamento de Engenharia de Materiais, Centro Federal de Educação Tecnológica de Minas Gerais, Belo Horizonte, Minas Gerais, Brasil. 


\section{INTRODUÇÃO}

O processo de Nitretação por Descargas Elétricas (NDE) propõe a adição de nitrogênio na superfície de materiais metálicos pelo método de implantação iônica, utilizando o canal de plasma formado durante a abertura do arco elétrico entre dois eletrodos, um a ferramenta e o outro a peça na máquina de eletroerosão por penetração [1].

A Usinagem por Descargas Elétricas (EDM) consiste no mecanismo de erosão do material, primeiramente, pelo uso da energia elétrica e modificado internamente para a energia térmica, completando uma série de descargas elétricas discretas, ocorrendo entre o eletrodo e a peça imersos em um fluido dielétrico. A energia térmica gera um canal de plasma entre o catodo e anodo com temperaturas elevadíssimas, iniciando substancialmente o aquecimento e a fusão na superfície de cada polo. Quando a pulsação direta da corrente é interrompida, o canal de plasma entra em colapso e causa redução repentina da temperatura, permitindo a circulação do dielétrico, retirando o material fundido do canal de plasma transportando-o em forma de partículas microscópicas. Após uma descarga, ocorre a formação de uma cratera e parte do material fundido deposita-se na superfície da cratera, devido à tensão superficial e efeitos de resfriamento, formando uma camada ao longo de toda superfície usinada, denominada pelos pesquisadores de camada refundida. Esta camada geralmente é frágil e apresenta trincas e poros, sendo necessário ser removida. Abaixo desta camada fica a zona afetada pelo calor (ZAC), que é apenas parcialmente afetada pelas altas temperaturas. As durezas da camada refundida e da ZAC dependem dos parâmetros de EDM, da capacidade de condução térmica e do próprio material [2].

A distância entre o eletrodo e a peça é preenchida pelo fluido dielétrico, bombeado e filtrado de um reservatório até a região de usinagem. O fluido dielétrico tem papel importante no processo EDM: refrigerar a região de usinagem, conduzir as partículas removidas e controlar a potência de abertura da descarga [3].

Os eletrodos mais eficientes para a aplicação são os que possuem alta condutividade elétrica e alto ponto de fusão. Os materiais mais utilizados no processo de EDM são o cobre eletrolítico e a grafita.

As ligas de titânio possuem características como: alta resistência específica, à corrosão e a fadiga, biocompatibilidade e boas propriedades criogênicas. Porém, as mesmas têm alto coeficiente de atrito, baixa resistência aos mecanismos de desgaste abrasivo e adesivo.

A aplicação do processo de EDM em ligas de Ti6Al4V com uma solução de ureia em água destilada, para verificar a formação de nitretos de titânio foi estudada por alguns pesquisadores (TiN) [4].

O objetivo deste trabalho foi avaliar a usinabilidade e nitretação por EDM da liga Ti6Al4V com solução de água e ureia, relacionada aos parâmetros: taxa de remoção de material (TRM), taxa de desgaste (TD) e desgaste volumétrico relativo (DVR); medir a espessura da camada nitretada por EDM via microscopia óptica; aferir o valor 
da microdureza da camada nitretada. Além de identificar os nitretos formados na camada nitretada por meio de difração de raio-x.

\section{MATERIAIS E MÉTODOS}

Foram utilizados nesta pesquisa eletrodos ferramentas de grafita e cobre no formato cilindro com $22 \mathrm{~mm}$ de diâmetro e $50 \mathrm{~mm}$ de comprimento. Foram fabricadas amostras (eletrodo peça) da liga de titânio Ti6Al4V com diâmetro de $19 \mathrm{~mm}$ e $13 \mathrm{~mm}$ de comprimento, ambos confeccionados por usinagem convencional. Os eletrodos de grafita foram secos em forno a temperatura de $300{ }^{\circ} \mathrm{C}$ durante 3 horas, antes de cada pesagem. Os eletrodos ferramentas e as amostras foram pesados em uma balança analítica digital com precisão de 0,0001 gramas, modelo MARK M antes e após a nitretação por descargas elétricas.

Foi utilizada no processo de NDE a máquina de eletroerosão por penetração, modelo Servspark Eletroplus EDM-540 com adaptações para possibilitar a realização dos experimentos. Para evitar a contaminação do fluido dielétrico, uma cuba auxiliar em aço inox AISI 304, foi posicionada no interior da cuba principal da máquina de EDM. Para permitir a limpeza da fenda de trabalho, foi instalada na tampa da cuba auxiliar uma bomba centrífuga com 120 w de potência, $3100 \mathrm{rpm}$, vazão de $51 \mathrm{l} / \mathrm{min}$ e pressão de 2 mca. A Figura 1 ilustrada as adaptações realizadas na máquina de EDM.

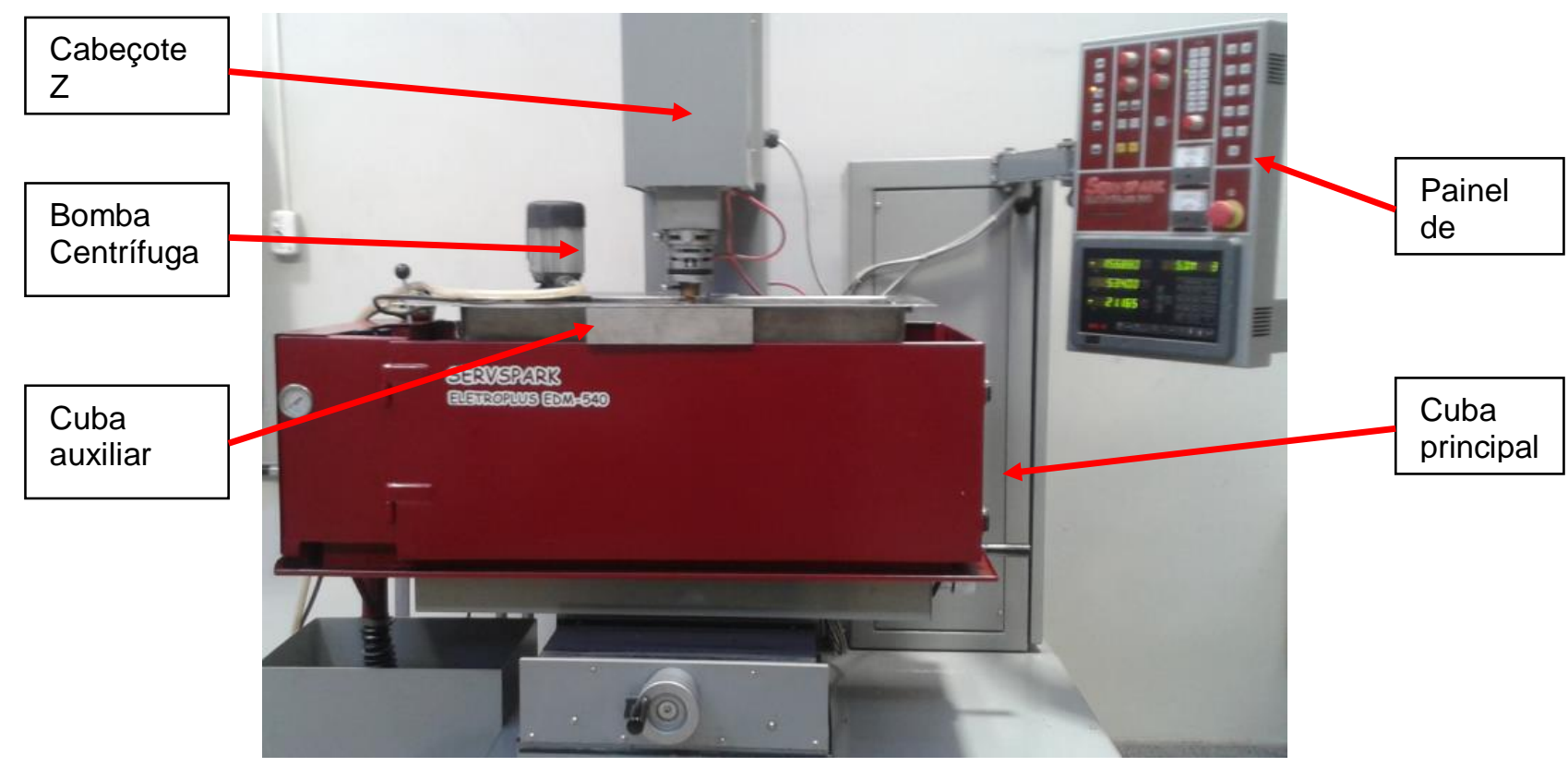

Figura 1. Máquina de eletroerosão adaptada para aplicação do processo NDE.

Foi utilizada solução aquosa como fluido dielétrico, formada com água deionizada e ureia farmacológica granulada na concentração de 20 gramas por litro. A condutividade inicial da água deionizada medida antes de formar o fluído dielétrico foi de $1 \mu \mathrm{S} / \mathrm{cm}$. A condutividade do fluído dielétrico foi aferida por meio de condutivímetro portátil com faixa de operação de 0 a $1999 \mu \mathrm{S} / \mathrm{cm}$ e incerteza de $\pm 2 \%$ da faixa de indicação, antes e após os ensaios.

A Tabela 1 apresenta os valores de condutividade da solução aquosa antes e após os experimentos. 
Tabela 1. Valores de condutividade do fluido dielétrico antes e após os testes.

\begin{tabular}{|c|c|}
\hline Fase & Condutividade $(\mu \mathrm{S} / \mathrm{cm})$ \\
\hline Início dos testes & 10 \\
\hline Final dos testes & 18 \\
\hline
\end{tabular}

Para fixação das amostras e dos eletrodos ferramentas foram utilizados porta amostras e porta ferramentas, respectivamente. A Figura 2 ilustra essa montagem no interior da cuba auxiliar.

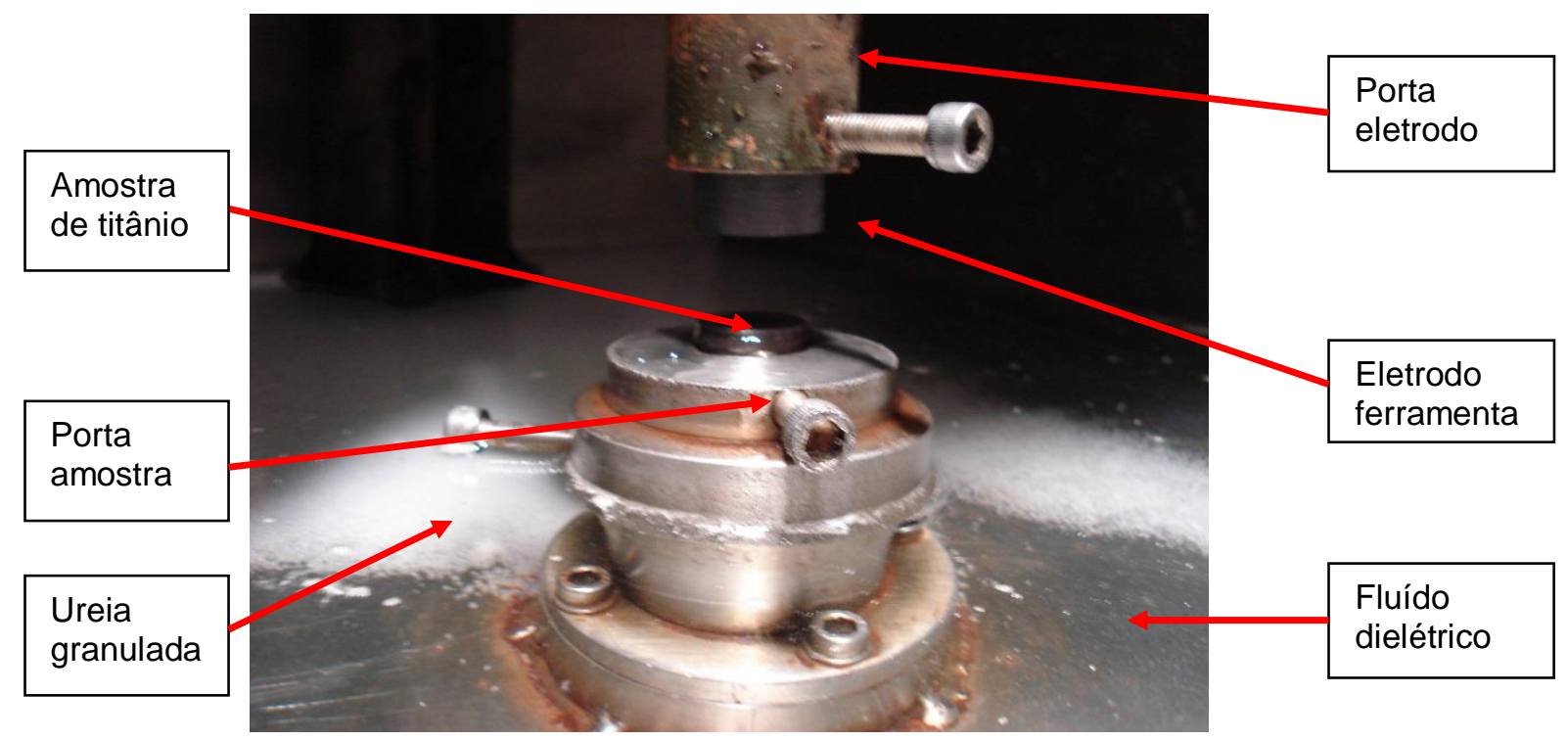

Figura 2. Posicionamento do eletrodo ferramenta e amostra de titânio no interior da cuba auxiliar.

Os parâmetros operacionais da máquina EDM foram ajustados para possibilitar a aplicação do processo NDE. A Figura 3 ilustra o painel para ajustes dos parâmetros.

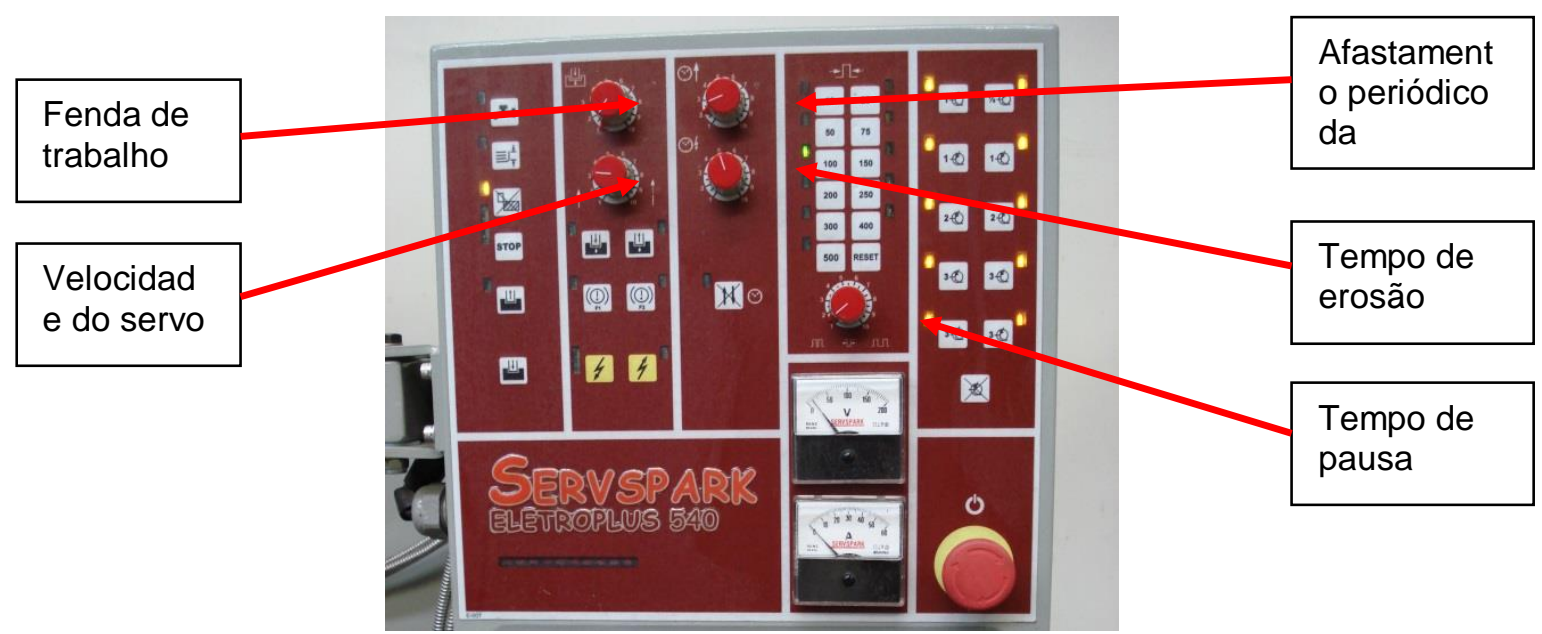

Figura 3. Painel de controle com indicação dos botões para ajustes dos parâmetros operacionais.

A Tabela 2 apresenta os parâmetros operacionais. O tempo de duração de cada teste foi de 10 minutos. 
Tabela 2. Parâmetros de trabalho ajustados na máquina de EDM.

\begin{tabular}{|l|c|}
\hline Polaridade do eletrodo ferramenta & Positiva $(+)$ \\
\hline Tensão & $110(\mathrm{~V})$ \\
\hline Corrente & $40(\mathrm{~A})$ \\
\hline Tempo de pulso & $100(\mu \mathrm{s})$ \\
\hline Tempo de pausa & $1,5^{\star}$ \\
\hline Fenda de trabalho & $1,0^{*}$ \\
\hline Velocidade do servo & $5,0^{*}$ \\
\hline Afastamento periódico da ferramenta & $3,0^{*}$ \\
\hline Tempo de erosão & $5,0^{*}$ \\
\hline
\end{tabular}

*Esses parâmetros foram ajustados diretamente nos botões do painel de controle.

Após os testes, amostras de titânio foram seccionadas com aparelho cutoff, embutidas e preparadas para análise micrográfica da camada nitretada. A espessura da camada foi medida por meio microscópio óptico. Ensaios de microdureza Vickers com cargas de $10 \mathrm{gf} \mathrm{a} \mathrm{20s,} \mathrm{foram} \mathrm{realizados} \mathrm{via} \mathrm{microdurômetro} \mathrm{com} \mathrm{microscópio} \mathrm{óptico} \mathrm{e} \mathrm{câmera}$ digital acoplada ao equipamento para registo de imagens. Difratogramas foram obtidos por meio de difração de raio-x.

A variação de massa das amostras usinadas foi mensurada, para avaliação o desempenho da taxa de remoção de material (TRM) que caracteriza a eficiência da remoção do material durante a usinagem expressa em $\mathrm{mm}^{3} / \mathrm{min}$, por meio da Equação $1[5]:$

$$
T R M=\frac{\Delta m}{\delta \times t}
$$

onde: $\Delta \mathrm{m}=$ variação da massa; $\delta$ = peso específico; $\mathrm{t}=$ tempo de usinagem.

A da taxa de desgaste (TD) apresenta o volume de material removido do eletrodo ferramenta por tempo $\left[\mathrm{mm}^{3} / \mathrm{min}\right]$. A relação entre o desgaste da ferramenta e o da peça dada pela Equação 2, fornece o valor do desgaste volumétrico relativo (DVR), expresso em valores percentuais, caracteriza o desempenho do processo de NDE [6].

$$
D V R=\frac{T D}{T R M} \times 100[\%]
$$

\section{RESULTADOS E DISCUSSÃO}

A Figura 4 apresenta imagem via microscopia óptica da seção transversal de amostra nitretada com eletrodo ferramenta de cobre capturada com câmera digital acoplada ao microdurômetro. 

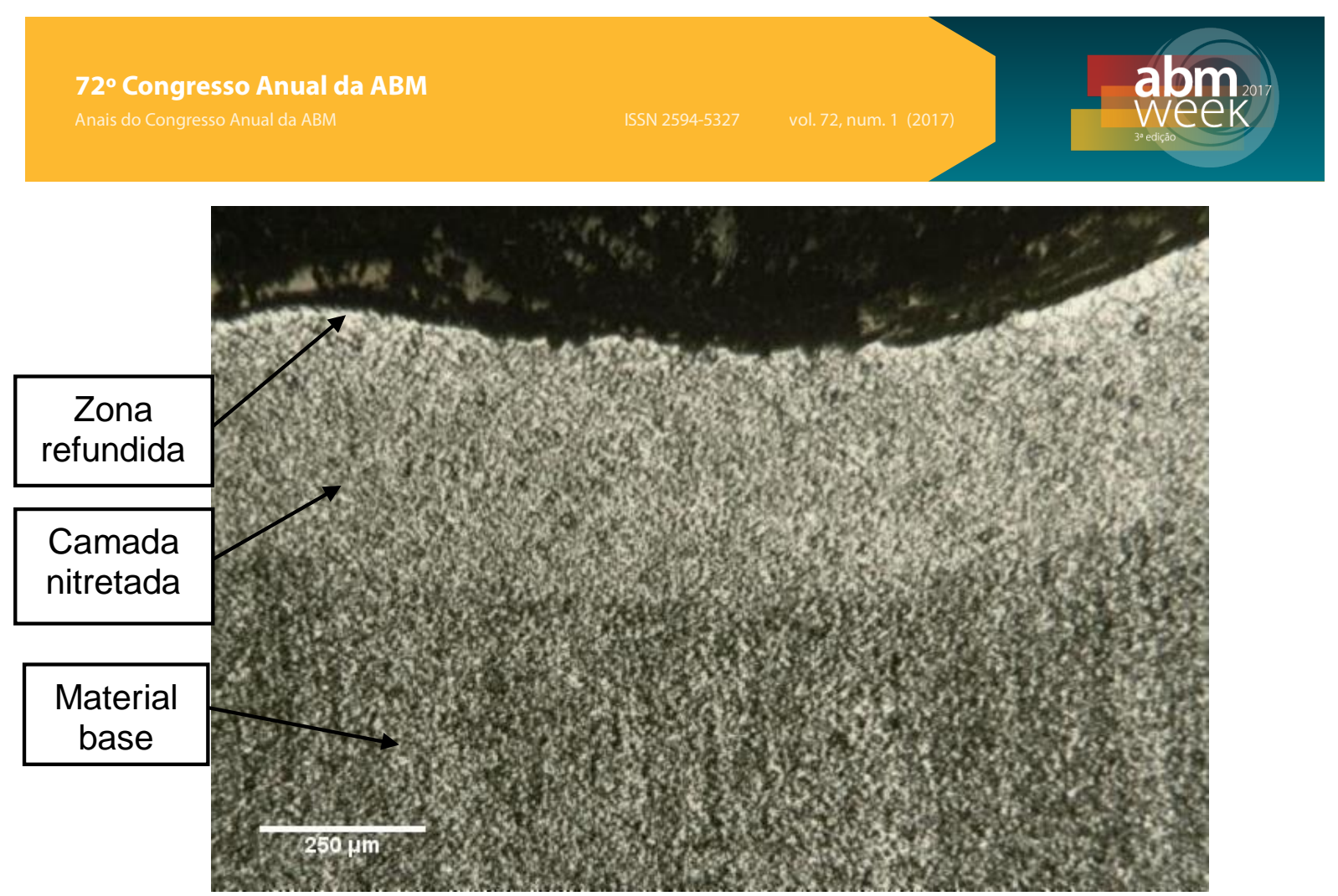

Figura 4. Microscopia óptica com ampliação de $100 \mathrm{X}$ de uma amostra de Ti6Al4V nitretada com eletrodo de cobre.

Observa-se que a camada nitretada mede aproximadamente $300 \mu \mathrm{m}$.

A Figura 5 apresenta imagem via microscopia óptica da seção transversal de amostra nitretada com eletrodo ferramenta de grafita capturada com câmera digital acoplada ao microdurômetro.

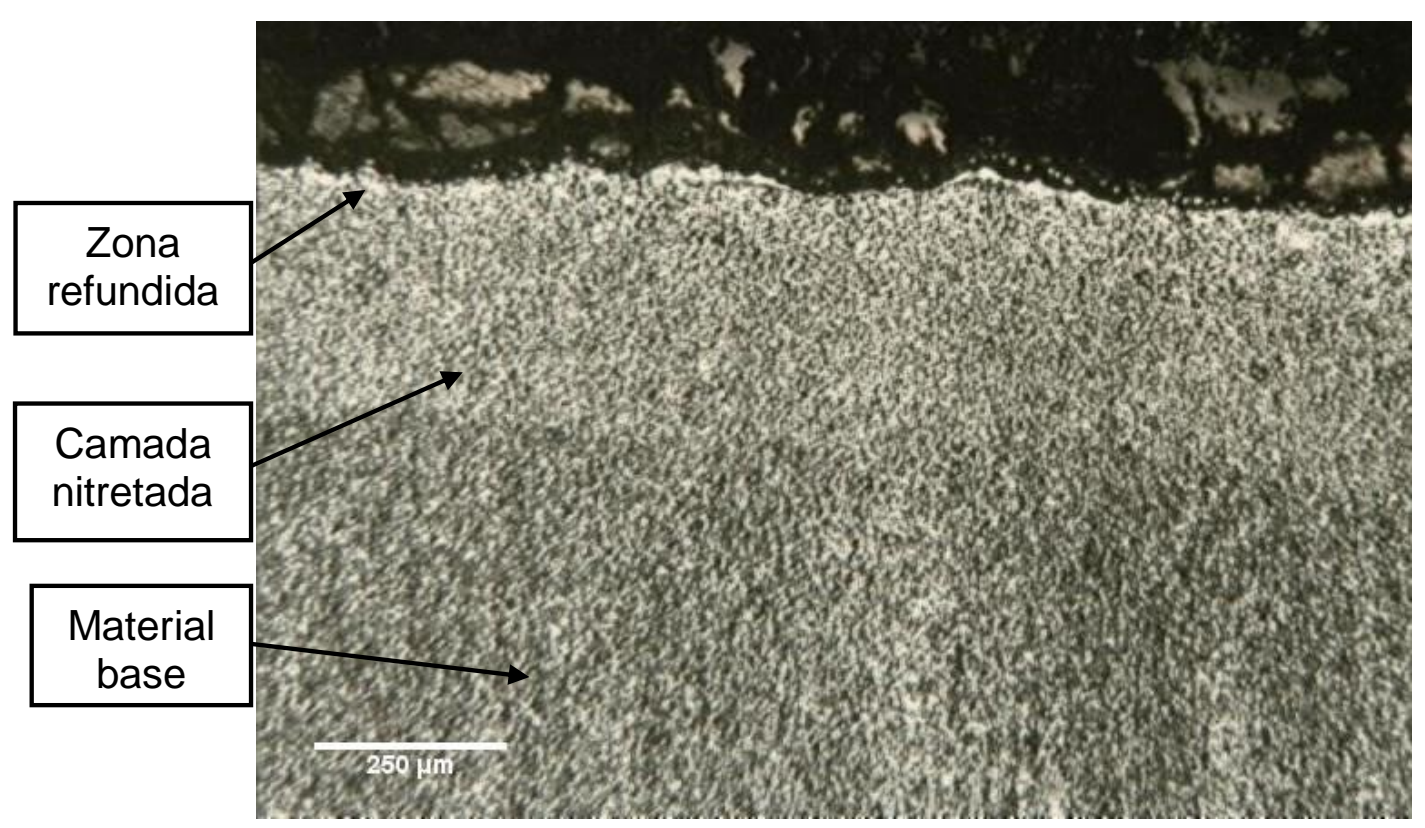

Figura 5. Microscopia óptica com ampliação de $100 \mathrm{X}$ de uma amostra de Ti6Al4V nitretada com eletrodo de grafita. 
Observa-se que a camada nitretada mede aproximadamente $250 \mu \mathrm{m}$. Os valores das espessuras da camada nitretada na liga Ti6Al4V utilizando eletrodo de cobre e de grafita foram similares, não apresentando grande discrepância entre suas espessuras.

A Tabela 3 apresenta os resultados de microdureza Vickers da camada nitretada com eletrodo ferramenta de cobre e de grafita e do material de base Ti6Al4V. Observa-se um ganho considerável nos valores de dureza das amostras após o processo NDE.

Tabela 3. Valores de microdureza Vickers do material base Ti6Al4V e das amostras nitretadas com eletrodo ferramenta de cobre e grafita, carga de $10 \mathrm{gf}$ a 20 segundos.

\begin{tabular}{|c|c|c|c|}
\hline \multirow{2}{*}{$\begin{array}{c}\text { Condição do } \\
\text { material }\end{array}$} & $\begin{array}{c}\text { Material base } \\
\text { Ti6Al4V }\end{array}$ & \multicolumn{2}{|c|}{ Camada nitretada (NDE) } \\
\cline { 3 - 4 } & Eletrodo de cobre & Eletrodo de grafita \\
\hline Dureza (HV) & $296,2 \pm 7,5$ & $587,5 \pm 41,9$ & $562,7 \pm 45,5$ \\
\hline $\begin{array}{c}\text { Ganho no valor da } \\
\text { dureza HV }\end{array}$ & - & $98,3 \%$ & $89,9 \%$ \\
\hline
\end{tabular}

A Tabela 4 apresenta os resultados das médias e desvios padrões para TRM, TD e DVR referente ao processo de NDE utilizando eletrodos ferramentas de cobre e grafita.

Tabela 4. Resultados do desempenho do processo NDE utilizando eletrodos ferramentas de cobre e grafita.

\begin{tabular}{|l|c|c|c|}
\hline Eletrodo ferramenta & TRM ( $\left.\mathbf{m m}^{\mathbf{3}} / \mathbf{m i n}\right)$ & TD ( $\left.\mathbf{m m}^{\mathbf{3}} / \mathbf{m i n}\right)$ & DVR (\%) \\
\hline Cobre & $23,66 \pm 0,48$ & $1,13 \pm 0,04$ & $4,78 \pm 0,14$ \\
\hline Grafita & $11,47 \pm 2,32$ & $3,7 \pm 0,45$ & $33,19 \pm 7,39$ \\
\hline
\end{tabular}

Comparando-se os valores de desempenho do processo NDE observa-se que a aplicação de eletrodo ferramenta de grafita apresenta maior desgaste volumétrico relativo em relação ao processo utilizando eletrodo de cobre. O comportamento da usinagem com eletrodo de grafita foi diferente do que ocorre na usinagem do aço. Geralmente a TRM é maior no aço quando se usa o eletrodo de grafita, comparado ao cobre.

A Figura 6 apresenta o gráfico intensidade versus ângulo de varredura $(2 \theta)$ com os resultados do ensaio de difração de raio-x, representando os difratogramas das amostras nitretadas com eletrodos ferramenta de cobre e grafita comparados com 0 material base Ti6Al4V. 


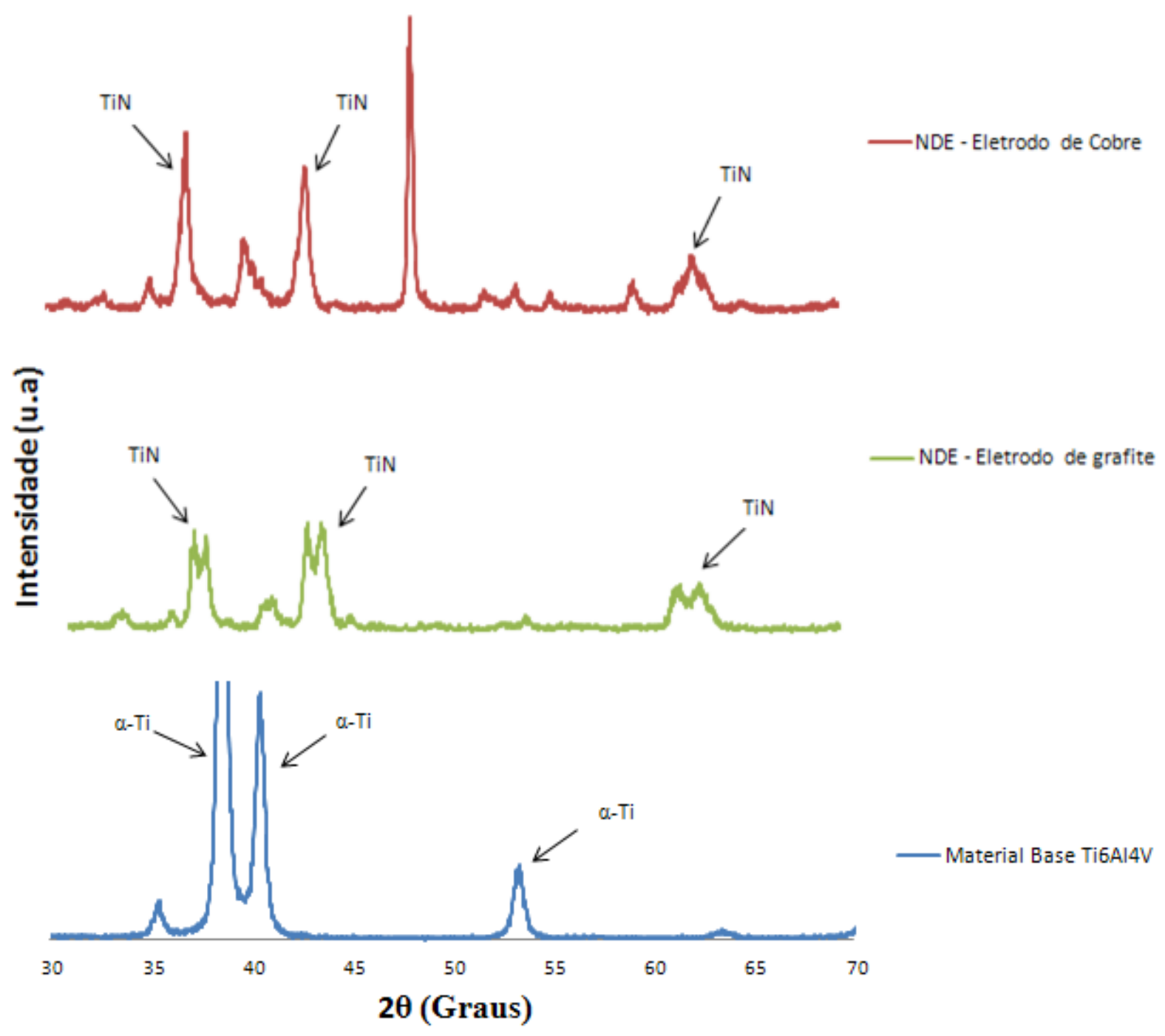

Figura 6. Difratograma das amostras nitretadas com eletrodos de cobre e grafita versus material base Ti6Al4V.

Observa-se nos espectros de varredura a presença de nitreto de titânio (TiN) nas superfícies das peças usinadas por NDE utilizando eletrodo ferramenta de cobre a $37^{\circ}, 43^{\circ}$ e $63^{\circ}$ bem como de grafita a $37^{\circ}, 44^{\circ}$ e $63^{\circ}$.

\section{CONCLUSÃO}

Foi observada a formação de uma camada nitretada nas superfícies usinadas no processo NDE com eletrodos ferramenta de cobre e de grafita.

A espessura da camada nitretada com eletrodo de cobre foi de aproximadamente 300 $\mu \mathrm{m}$ enquanto que para eletrodo de grafita foi de aproximadamente $250 \mu \mathrm{m}$.

Os ensaios de microdureza Vickers demonstraram um ganho considerável, aproximadamente $90 \%$, nas amostras usinadas no processo NDE em relação ao material base.

O desempenho de usinagem NDE utilizando eletrodo ferramenta de grafita promove maior desgaste volumétrico relativo comparado ao processo com eletrodo de cobre. 
Por meio dos resultados do ensaio de difração de raio-x foi possível evidenciar a formação de nitretos de titânio caracterizando a camada nitretada.

\section{Agradecimentos}

Ao CEFET MG pelo apoio para participação e apresentação do trabalho no congresso.

\section{REFERÊNCIAS}

1 Raslan AA, Santos RF, SILVA ER. Método de Nitretação através da Usinagem com descargas elétricas-NDE. FAPEMIG.2012.

2 Mcgeough JA. Advanced methods of machining. London: Chapman and Hall, 128-152; 1988.

3 Fuller JE. Electrical Discharge Machining. Metals Handbook. 16: 557-564; 1989.

4 Yan BH, Tsai HC, Huang FY. The effect in EDM of a dielectric of a urea solution in water on modifying the surface of titanium. International Journal of Machine Tools \& Manufacture. 2005; 45:194-200.

5 Santos CE. Influência da corrente elétrica no processo de nitretação por descargas elétricas do AÇO AISI H13. [dissertação de mestrado]. Belo Horizonte: Centro Federal de Educação Tecnológica de Minas Gerais; 2015.

6 Amorim FL. Tecnologia de eletroerosão por penetração da liga de alumínio AMP 8000 e da liga de cobre. CuBe para ferramentas de moldagem de materiais plásticos [tese de doutorado]. Florianópolis: Universidade Federal de Santa Catarina;2002. 